\title{
GENERO Y POLITICAS DE SALUD DE LA MUJER EN AMERICA LATINA: caso Perú (PARTE I) *
}

\author{
Marina Violeta Estrada Pérez de Martos \\ Rosa Maria Godoy Serpa da Fonseca ***
}

\begin{abstract}
MARTOS, M.V. E. P. de; FONSECA, R.M.G.S. da Genero y politicas de salud de la mujer en America Latina: caso Perú (Parte I). Rev.Esc.Enf.USP, v.30, n.1, p.44-57, abr. 1996.

En este estudio se hace una presentación y análisis de la problemática de la mujer, considerando su condición social, de acuerdo a la ideologia androcentrista en la sociedad. Se hace una breve evolución histórica de la mujer bajo el recorte analítico de género. De otro lado, se analiza las políticas públicas y de salud de la mujer en América Latina, especialmente en el Perí, desde el Incanato, explicando las razones de la formulación e implantación del Programa de Planificación Familiar y del Programa de Atención Materno Infantil.
\end{abstract}

UNITÉRMINOS: Ideología. Género. Planificación familiar. Políticas de salud.

\section{GENERALIDADES}

La salud de la mujer no puede ser analizada en forma siple y aislada de su ubicación a través de la historia. s necesario relacionarla con el contexto socio-económico cultural y político en el cual la mujer se deenvuelve, por estar directamente relacionado como determinante del proceso saludenfermedad.

La definición de la Organización Mundial de la Salud (OMS), tal como lo sustentan DALLARI (1988) y MAMEDE (1989), nos ayuda a analizar la

\footnotetext{
* Basado en la monografia presentada en la Disciplina Temas Avançados en Salud Colectiva del Programa de Post-grado en Enfermería de la Escuela de Enfermería de la Universidad de São Paulo - Brasil (EEUSP)

* Enfermera. Doctoranda del Programa de Post-grado en Enfermería. EEUSP. Docente de la Escuela Académico Profesional de Enfermería de la Facultad de Ciencias de la Salud de la Universidad Nacional de Cajamarca (Perú).

*** Enfermera. Doctora en Enfermería. Docente y orientadora del Programa de Post-grado en Enfermería - EEUSP
} 
situación dentro del contexto mencionado, es decir, dentro del contexto del mundo en que vivimos, considerandola además como um derecho humano ${ }^{(1)}$; "Salud es el estado de completo bienestar, tanto físico, mental y social y no solamente la ausencia de enfermedad". Sin embargo, la salud como fenómeno aislado prácticamente se constituye en algo utópico e imposible por las desigualdades sociales, económicas, de género, entre otras, que discriminan segmentos grandes de la población entre los que se encuentran las mujeres, y más aún si son pobres, de edad avanzada, de color o indígenas.

Los roles asignados históricamente a las mujeres en las sociedades ocidentales han sido siempre los de esposa, madre, ama de casa, circunscribiendolas prácticamente al ámbito del hogar. Muy diferente a los roles del hombre que los direccionan al ámbito público; desligandolos completamente de la responsabilidad de los quehaceres domésticos y de la crianza de los hijos, por el hecho de que él debe dedicarse al mantenimiento económico de la familia como rol innato a su persona - género - tal como es manifestado por COSTA (1992) "... de hecho hay una causa diferente del porque del trabajo entre hombres y mujeres, el hombre trabaja porque es hombre y debe mantener la familia, la mujer en cambio solo para ayudar cuando el salario del marido es insuficiente".

No obstante, es necesario resaltar que el mantenimiento de la familia le significa al hombre lidiar con una serie de situaciones que dependen de su forma de inserción en el sistema productivo y de la clase social a la que pertenece, lo cual acarrea indudablemente desigualdades sociales profundas en la población. Esta afirmación es fundamentada a través del concepto filosofico materialista histórico y dialéctico de clases sociales, el cual es enunciado de la siguiente manera:

"Las clases sociales son grandes grupos de hombres que se diferencian entre si por el lugar que ocupan en el sistema de producción históricamente determinado, por las relaciones en que se encuentran frente a los medios de producción (relaciones que en gran parte son establecidas y formuladas por leyes), por el papel que desempeñan en la organización social del trabajo y, consecuentemente, por el modo de producción por el cual reciben la parte de la riqueza social que les cabe. Las clases son grupos humanos en los cuales uno puede apropiarse del trabajo del otro, por ocupar puestos diferentes en un régimen determinado de economía social". (LENIN apud FONSECA, 1990)

Por otro lado si se analiza la situacion del hombre en relación a sus derechos de acuerdo al concepto de ciudadanía implica que los derechos del hombre "no se originan en un código de valores políticos, sino en un sistema de estratificación ocupacional definidos por norma legal”. (SANTOS, 1979)

(1) La Constitución adoptada por la Conferencia Internacional de la Salud, realizada en Nueva York del 19 al 22 de Julio de 1946 y firmada el 26 de Julio de 1946 por representantes de 61 Estados, presenta esta definición como el primer principio básico para la "felicidad, las relaciones armoniosas y la seguridad de todos los pueblos"

Rev.Esc.Enf.USP, v.30, n.1, p.44-57, abr. 1996. 
Retomando el análisis histórico de la problemática femenina, se observa que la mujer a diferencia del hombre no sólo es discriminada en el aspecto social, sino en todos los aspectos, por el hecho de que ella es considerada por la sociedad como un ser inferior, sin ninguna o pocas posibilidades, presentandose esto desde tiempos muy remotos. Por ejemplo, según VERUCCI (1987) para ARISTOTELES, "la mujer es mujer en virtud de ciertas carencias de cualidades" y para SANTO TOMAS DE AQUINO, "la mujer es un hombre incompleto, un ser ocasional".

Existen muchas definiciones y estudios que demuestran que existen prejuicios sexuales, y además, afirman y refuerzan la condición de desigualdad de la mujer dentro de la sociedad, comprendiendose así la opresión y discriminación de que es objeto y víctima la mujer. "A pesar de que en los últimos aÑos se han operando algunos cambios, todavía persiste la concepción de roles tradicionales, por lo que tal realidad coloca a las mujeres en una situación de desigualdad en cuanto a oportunidades y derechos fundamentales como son educación, acceso al empleo sin discriminación, salarios equitativos, participación política, acceso a cargos donde se da la toma de decisiones, así como el ejercicio y goce de la sexualidad, por citar algunos" (ROMERO, 1988).

Sería del caso que la situación de la mujer sea discutida más amplia y profundamente, al igual que sus roles con relación al hombre, fundamentando la diferencia en base al género y no basandose estrictamente en el aspecto biológico o sexual como se ha venido manejando el tema a través de la historia.

Muchos autores analizan la diferencia de roles entre hombres y mujeres basandose en el sexo, y a partir de ello explican la subordinación femenina en términos "naturales e inevitables", y aún más centrandose exclusivamente en la función reprodutiva, tal como lo manifiestan algunos autores citados por ROMERO (1988).

Para ERIKSON, "El diseño somático de la mujer abriga un espacio interior destinado a dar luz a la progenie de los hombres elegidos y con el un compromiso biologico, psicologico y ético para cuidar de la infancia humana".

WEININGER, por su vez manifiesta que "La mujer se consume en la vida sexual en la espera de la cópula y la multiplicación, es decir, en sus relaciones como esposa y madre... la mujer no es otra cosa que sexualidad; el hombre es un ser sexual pero también es algo más... las mujeres no tienen existencia, ni esencia, son la nada. Se es hombre o mujer, según se sea o no se sea".

FREUD (apud MAMEDE, 1989) igualmente colabora diciendo que "la anatomia es destino".

Por el contrario si el análisis de la situación femenina se lo hace en base al género, permitiría enfocar el tema de una optica más amplia y relacionandola con el proceso de socialización, el cual es responsable de la formación, mantenimiento y transmisión de los valores, creencias y actitudes que van a influir en el modo de pensar de la gente. 
Segun BLEICHMAR (apud ROMERO, 1988), "género es la categoria donde se agrupan los aspectos psicologicos, sociales y culturales de la femenidad/masculinidad, reservandose sexo para los componentes biologicos, anatómicos..."

COSTA (1992) reafirma lo anteriormente manifestado diciendo: “... el sexo trajo muchas y fáciles asociaciones con la biología (...); la elaboración del concepto - género - vió enfrentar esta dificultad, procurando mostrar como la condición del hombre y de la mujer es socialmente definida".

Los roles del género femenino obedecen ciertamente, a una ideología dominante en la sociedad, una ideología eminentemente patriarcal; tal como lo expresan los siguientes autores:

LOPES (1988) - "La sociedad patriarcal propone concretamente la manera como se discrimina, limita y restringe la libertad de la mujer".

PINOTTI (1988) - "Las diferencias entre las actividades del hombre y la mujer son secundarias y no primarias, adquiridas y no innatas".

BEAUVOIR (1981) - "No se nace mujer, una se vuelve mujer (...) Ningún destino biológico, psíquico o económico define la forma que la fémina humana asume en el seno de la sociedad, es el conjunto de la civilización que elabora ese producto".

Es decir, toda la influencia recibida por la mujer desde el momento de su nacimiento prácticamente la predisponen para los roles que la sociedad le tienen designados con muchísima anticipacion, reafirmando la concepción de roles tradicionales de madre, esposa y ama de casa.

La situación más objetiva de discriminación de la mujer que concretamente se percibe es en el trabajo, la doble jornada que la mujer realiza - permanencia del trabajo en el hogar, junto con el rol de ser madre y esposa - y la esfera pública, es la más evidente manifestación de discriminación en cuanto a horas de trabajo, tareas, salarios, posibilidades de ascenso, entre otras. STOLCKE (apud LOPES, 1988), indica: "a pesar de la incorporacion en el trabajo asalariado, la responsabilidad principal de las mujeres continúa siendo tener hijos y crialos y las tereas relacionadas a esto".

Igualmente, la división social del trabajo dado a nivel de sexualización de las ocupaciones redunda en la discriminación. PINOTTI (1988) así lo manifesta: "La cultura y el estilo de vida de un pueblo, sus instituciones, usos y costumbres son los que acavan por determinar la división del trabajo entre sexos".

Además, las mujeres que trabajan geralmente lo hacen bajo la dirección del hombre o deben cumplir en muchos casos tareas de muy bajo prestigio social; "así, el trabajo femenino tiene actualmente una doble subalternidad, en el hogar y fuera de el; es más barato, igualmente apto; menos reinvindicativo e igualmente sumiso" (LOPES, 1988). 
Por otro lado, el modelo de produción capitalista, como en todas las situaciones, se aprovecha y mantiene por conveniencia la subalternidad de la mujer.

"El modo de producción capitalista que si no engendro la dominación entre sexos, legitima, usa y mantiene la desigualdad en todos los niveles para consolidar su propósito... El capitalismo procesó innumerables mudanzas, pero conservo lo que da soporte a la estructura de las relaciones sociales de dominación. La creación de las condiciones subordinadas del trabajo de la mujer ha sido uno de los soportes del mantenimiento de las relaciones de producción". (LOPES, 1988)

Sin embargo, como se esperaría, la situación de la mujer no es diferente en los modelos socialistas donde, como manifiestan algunos críticos, no solamente persiste la desigualdad sexual sino que en algunos aspectos puede considerarse que las mujeres de los países socialistas están en peores condiciones que las mujeres de países capitalistas.

"Lejos de haber sido emancipadas como se manifiesta retóricamente, la carga de trabajo femenino se aumento, no habiendo una redefinición sustancial de las relaciones entre los sexos. A los papeles tradicionales de ama de casa y madre, se han sumado los de asalariada de tiempo completo y los de activista política..." (MOLYNEUX, 1984).

Es decir que mientras el hombre domine el poder y la producción, aún con concepciones ideologicas diferentes, continuará discriminando a la mujer para su propio beneficio. Concluyendo que "la exclusión de las mujeres de las actividades públicas y la construcción de un ideal femenino, asociado a las funciones en el interior de la familia delimitan los papeles legítimos de la mujer a aquellos directamente relacionados a la reproducción de la fuerza de trabajo, lejos de cualquier participación en los procesos decisorios en la esfera pública" (LABRA, 1989).

Cabe resaltar finalmente la violencia (1) contra las mujeres tanto sexual, física, psicologica y el de negarles los derechos humanos fundamentales como la vida, la libertad, la seguridad; por haber sido excluídas de la democracia y el desenvolvimiento tanto en el mundo público como privado. Por tanto es preciso, como recomienda BUNCH (s/d), que la mujer conozca la Declaracion Universal De Los Derechos Humanos, para saber todos los aspectos a los cuales tiene derecho sin discriminaciones de ningún tipo. Igualmente que "Nadie puede ser sometido a tortura, tratamientos o castigos crueles, deshumanos o degradantes", como sucede con millares de mujeres en el planeta.

\footnotetext{
(1) "Violencia" es fundamentalmente violación de la libertad y del derecho de alguien a ser sujeto constituyente de su propia historia. Queda claro que toda violación de género es violación de los derechos humanos (DORA, 1992).
} 


\section{LA HISTORIA DE LA MUJER EN LA SOCIEDAD}

La historia de la situación de la mujer no es suficiente para analizar su posición y status en la construcción de la sociedad, sólo se la usa para referir la localización del poder, la diferencia de papeles y posiciones de desigualdad que las mujeres tienen dentro de la sociedad.

La sumisión de la mujer es muy tangible durante toda la historia de la sociedad. Prácticamente fue el hombre quien dominó el poder y la producción durante todas las épocas.

Inicialmente en siglos pasados, en la era llamada del matriarcado, como la denominan ENGELS (1984), SALVATORE (1988) y TOCKUS (1986) (citados por LORENS, 1990), la mujer ocupaba un papel social en la producción de la familia. La función reproductiva del hombre era desconocida y el de las mujeres no se daba a costa de la sumisión de los hombres, razón por la cual actualmente estas sociedades son llamadas matrilineares. (LABRA, 1989)

"La forma más simple de organización familiar fue la mujer viviendo con sus hijos, viviendo juntos en clan (...) Conociendose la madre y no el padre, la herencia era trazada por la línea materna (...) elevando la importancia de la madre en el hogar y en la sociedad" (LEITE, 1994)

Para DURANT (apud LEITE, 1994) el paso hacia las sociedades patriarcales se dió cuando fue descubierta la función reproductiva del hombre y las condiciones para el surgimiento de la propriedad privada por la producción del excendente que era apropriado por los que manejaban los medios de producción.

"El crecimiento de la propiedad privada y transmissible bajo la forma de productos de la tierra, trajo consigo la subordinación sexual de la mujer, pués el marido exigía ahora la exclusividad de ella para ser el destino del fruto de su trabajo. El surgimiento del patriarcado fue fatal para la supremacía y autonomía de la mujer (...). Estaba definitivamente cerrado el período histórico en que la mujer respondía por la unidad familiar, por la organización de la sociedad y por la herencia de los bienes". (LEITE, 1994)

Durante todo el tiempo histórico las mujeres siempre fueron excluídas de las guerras, riquezas, leyes, artes, ciencia, gobierno; reconocidas todas estas actividades como propias de la civilización. Esta exclusión se dío aún en los períodos considerados de avance y progreso (KELLY, 1986):

- En Atenas, se aceptó el concubinato y el confinamiento de las esposas en el gineceo;

- En el Renacimiento, se domesticó a la esposa burguesa para la reproducción de la riqueza;

- En la Revolución Francesa, se persiguieron a las mujeres que 
atravesaban las líneas de demarcación de las clases, y expresamente se excluyeron a las mujeres de la libertad, igualdad y fraternidad.

En la Revolución Industrial, en el siglo XVIII, tanto hombres como mujeres se convirtieron en operarios; logrando la mujer reconquistar la importancia económica que tuviera en algún momento, no obstante sin lograr cambios en su condición de subalternidad.

Durante el siglo XIX, los procesos de urbanización, industrialización, inmigración y aculturación contribuyeron de manera decisiva en las alteraciones sufridas por la unidad familiar. En la urbanización específicamente se observó una disminución de la autoridad paterna y una mayor participación de la mujer en las actividades lucrativas; pero no se alteraron ciertas características feudales como la tolerancia del adulterio masculino e intolerancia del femenino, tabú contra la pérdida de la virginidad de la mujer, etc. (VERUCCI, 1987).

Después de la segunda guerra mundial, la mujer conquistó pequeÑos espacios en los frentes de actuación; sin embargo en 1945, en la Organización de las Naciones Unidas (ONU) reconocen que en más de un tercio de los Estados la mujer no tenía derecho al voto, siendo que en Nueva Zelandia ese derecho la mujer lo había conquistado en 1893.

De otro lado, la Organización Internacional del Trabajo (OIT) hasta ese momento no discutía aún el derecho de la mujer al trabajo y a la igualdad de salarios. Mas en 1951, La Conferencia Internacional de la OIT aprobó el Convenio respecto a la igualdad de remuneraciones entre la mano de obra masculina y la mano de obra femenina, por un trabajo de igual valor; y en 1958, la misma Conferencia aprobó el convenio respecto a la discrimiación en materia de empleo (PAREJA, 1986).

\section{EVOLUCION HISTORICA DE LAS POLITICAS PUBLICAS Y DE SALUD EN EL PERU DESDE EL IMPERIO INCAICO HASTA LA SEGUNDA GUERRA MUNDIAL}

La República del Perú cuenta con una población estimada de 22 millones de habitantes en 1989, los cuales residen en su mayoría en zona rural (65.2\%). Tiene una área aproximada de 1,285.216 Km² (BERTOLOZZI; SHIMA; EGRY, 1990 ), con tres regiones muy marcadas con características geofísicas de Costa, Sierra y Selva. Los productos que se exportan son el pescado, algodón, madera, café, minerales y petróleo. El Perú se divide actualmente en once regiones debido al proceso reciente de regionalización y descentralización, como una estrategia para lograr algunas transformaciones del Estado. Cada región está conformada por uno o dos departamentos o sub-regiones de acuerdo a sus características de semejanzas, sobre todo geofísicas. Los departamentos o 
sub-regiones se dividen en provincias y estas a su vez se dividen en distritos (MINISTERIO DE SALUD, 1990). La población económicamente activa (PEA) es de cerca de $51.9 \%$ de los cuales $41.4 \%$ pertencen al sector primario, $15.7 \%$ al sector secundario y $42.9 \%$ al terciario (BERTOLOZZI; SHIMA; EGRY, 1990).

La situación de la mujer peruana no difiere mucho de las mujeres de otros países, sobre todo de aquellas de los llamados países del tercer mundo a los que pertenece el Perú. El manejo y tratamiento de la situación de la mujer tiene algunas variantes de acuerdo al momento histórico que vivió el país, y de acuerdo al lugar de origen de cada mujer, sea de zona urbana o rural, sea de costa sierra o selva.

Si se rotrocede la historia inclusive hasta situarnos en las raíces de origen del Perú, encontramos que en el Imperio Incaico se mantuvo una organización extremadamente elevada.

"El objetivo fundamental para sus gobernantes era el hombre a quien garantizaban la atención de sus necesidades desde la cuna hasta la tumba, al igual que en las sociedades socialistas del presente" (VEGAS, 1989).

El Imperio Incaico fue una sociedad autárquica que no conoció el hambre debido al sentido de previsión existente logrando la "felicidad" de sus componentes por su ejemplar administración de justicia, sus valores morales y el sentido de solidariedad humana que desplegaron. Sus lemas principales eran: "No robes (Ama Sua), No mientas (Ama Llulla) y No seas perezoso (Ama Kella)". (VEGAS, 1989)

No obstante estas características, en la sociedad incaica la mujer ocup6 un lugar de subalternidad y sumisión, tanto ante el Inca Jefe, considerado como un "Dios", como ante el marido a quien le pertenecía. Sin embargo, a pesar de mantenerse esta desigualdad, la mujer además de desarrollar las tareas domésticas, participó en la agricultura, artesanía, etc., es decir, tuvo un papel importante en la producción y desarrollo de su comunidad.

Con la llegada de los conquistadores españoles, la situación del Imperio Incaico cambi6 bruscamente en todos los sentidos, ya que los españoles valiendose del "engaño, de la mentira, de la traición y de la crueldad" que fueran las primeras lecciones que recebieron los antiguos peruanos de la civilización europea. (VEGAS, 1989). Asi, los "indios" fueron despojados de sus bienes y riquezas, de sus tierras, familia y sobre todo de su cultura.

"Los conquistadores no se ocuparon casi sino de dis tribuirse y disputarse el pingüe botín de guerra. Despojaron los templos y los palacios de los tesoros que guardaban; se repartieron las tierras y los hombres sin preguntarse siquiera por su porvenir como fuerzas y medios de producción." (MARIATEGUI, 1984)

Los incas pasaron a ser dominados oprimidos y discriminados, siendo tratados por los españoles, desconocedores absolutos de la Cultura Incaica, 
"peor que animales", por ser utilizados como "bestias de carga" para acumular la riqueza - capital - para ser llevada hacia Europa.

"... a medida que se solidificaba la dominación, se intensificaba la explotación inhumana de los naturales a quienes se hacia trabajar en las minas y los obrajes hasta el vómito de sangre de su tuberculinización. Al indio muerto automáticamente se le remplazaba por otro joven y sano." (VEGAS, 1989)

"La población indígena, que quizá bordeaba los 15 millones en 1525 , no pasaba de un millón y medio en 1571." (ARANIBAR, s/d)

Si para el hombre fue sumamente difícil vivir en esta época, para la mujer la situación se tornó insoportable ya que, además de servir como esclava en el trabajo, ella fue violada, torturada y humillada. Cuando los españoles invasores tenían deseos de satisfacer su apetito sexual, lo hacían utilizando la mujer "india", las cuales, como manifiesta ROLDAN, eran consideradas "iguales o inferiores a los animales" Los españoles utilizaban a las mujeres comportandose como "bestias" porque, como plantea el mismo autor "... incluso el animal cuando desea poseer la hembra, lo hace con cierto 'sentimiento', la corteja y la enamora; mas el invasor no manifiesta ningún tipo de halago ni consideración para con las nativas, las toman a la fuerza y sencillamente las violan, no uno solamente sino varios, muchos quizás." (ROLDAN, 1986)

Esto significó para la mujer india una situación insostenible porque para ella una relación sexual normalmente tenía una connotación "ríticomítico-religiosa" y de la "noche a la mañana" se tornó una "tortura humillante". (ROLDAN, 1986).

Podría decirse que a partir de esta época se instala un modelo de producción cuyo único objetivo era también como hoy, la acumulación para grupos o sociedades favorecidas muy distantes de nuestra realidad, en base a la esclavitud de los denominados "indios" y de esclavos negros que fueron traídos por los españoles.

Según MARIATEGUI (1984), la codicia fue la responsable para que los españoles se ocupen preferentemente en la minería, y convertir en un pueblo minero al que bajo el gobierno de los Incas y desde sus más remotos orígenes había sido un pueblo fundamentalmente agrario, lo cual origin 6 irremediablemente la necesidad de imponer al "indio" la dura ley de la esclavitud. "El trabajo del agro, dentro de un régimen naturalmente feudal, hubiera hecho del indio un siervo vinculado a la tierra. El trabajo de las minas y de las ciudades, debían hacer de él un esclavo." (MARIATEGUI, 1984)

Las relaciones de trabajo que se establecieron en ese entonces determinaron como sucedió en la gran mayoría de países de América Latina, la forma como se estructuraban las condiciones de vida y de salud de la población en esos diversos momentos históricos. (OLIVEIRA, 1988) 
La época de la esclavitud, que en la República del Perú fue abolida en 1863, por Don Ramón Castilla, Presidente de ese, entonces se caracterizó por ausencia de acciones para prevenir y recuperar la salud de la poblacion. La mortalidad principalmente infantil fue elavadísima debido a las calamitosas condiciones en las que eran obligados a vivir los esclavos.

Según PAREJA (1986), a principios del siglo XX las condiciones de trabajo que debieron soportar los obreros y empleados fueron extremadamente difíciles por no existir norm as que regulasen esta actividad. Las jornadas de trabajo para hombres, mujeres y niños se prolongaban hasta de diez a doce horas diarias e inclusive más, en establecimientos sin requisitos de higiene, seguridad, hipoventilados y mal iluminados, por tanto, expuestos a sufrir entre otros problemas, accidentes de trabajo y enfermedades ocupacionales.

Los salarios que obtenía la clase trabajadora eran muy bajos; no gozaban de estabilidad laboral, compensaciones ni indemnizaciones por tiempo de servicios. Bajo estas circunstancias la clase trabajadora fue obligada a vivir en deprimentes condiciones. La salud de la población se encontraba comprometida y propícia para el surgimiento de enfermedades infectocontagiosas entre las que sobresalieron la tuberculosis, viruela, malaria, peste, entre otras. En esta época las acciones de salud además de escasas fueron muy específicas, ya que solamente se dereccionaban al saneamiento ambiental, centrandose en las Ciudades Costeras sobre todo Lima, la Capital, y los puertos más importantes, entre los que se encontraban El Callao y Chimbote, a través de los cuales la economía peruana y el capitalismo extranjero, de carácter financiero-mercantil, mantenía sus relaciones comerciales con el exterior. (QUIJANO, 1985)

Caso semejante sucedió en Brasil, como lo manifiestan GERMANO (1985), ROSSI (1980), SILVA (1986), OLIVEIRA, (1988) "La Salud Pública estuvo direccionada inicialmente a las campañas de urbanización y saneamientos de puertos, con vista a garantizar la circulación y comercialización de los alimentos y productos en dirección a la Metrópoli". (OLIVEIRA, 1988)

La mujer como participante de la fuerza laboral representaba una pequeña cantidad de la población femenina total, por tanto, muy pequeña comparada a la participación masculina; y por lo general las actividades que realizaba eran de muy bajo pestigio social; "la población femenina es considerada y tratada como reprodutora y mantenedora de la fuerza de trabajo a través de sus labores en el hogar y en la reprodución de la especie" (PAREJA, 1988).

Por presión de la masa trabajadora, durante el primer gobierno de Don José Pardo y Barreda (1904 - 1908), se formularon diez proyectos sobre : higiene y seguridad de los trabajadores, trabajo de los niños y mujeres, descanso obligatorio, horas de trabajo de los hombres adultos, indemnización 
por accidentes de trabajo, entre otros. El proyecto sobre trabajo de mujeres y niños fue aprobado por el Congreso, pero no se formuló la ley sino hasta el segundo gobierno de Don José Pardo y Barreda (1915 - 1919), Ley n ${ }^{\circ} 2851$ del 23 de noviembre de 1918, la cual regulo entre otros aspectos la jornada de ocho horas de trabajo y cuarenticinco semanales, una hora de lactancia, sala cuna para los hijos menores de un año, indemnizaciones por despedida injustificada, por despedida dentro de los tres meses anteriores y posteriores al parto e indemnizaciones por accidentes de trabajo (PAREJA, 1986).

Es importante resaltar que la Legislación Peruana trat 6 a la mujer trabajadora de acuerdo a un régimen laboral específico, el cual cambió de acuerdo a la evolución de la sociedad, al punto de llegar a expresar según el Código Civil de 1984 en su artículo 291; "El trabajo del hogar y el cuidado de los hijos a cargo de uno de los cónyuges - sea este hombre o mujer - genera en el otro cónyuge la obligación de sostener la familia, sin excluir por ello la obligación mútua de ayuda y colaboración tanto en el hogar o fuera de el". (PAREJA, 1986)

Aparentemente es una disposición que posibilita la igualdad de género; sin embargo en la vida cotidiana no se efectiviza, ya que las labores domésticas son realizadas exclusivamente por la mujer como tareas inherentes a su género; aún cuando trabaje fuera del hogar. El trabajo doméstico no es considerado importante por no influir "aparentemente" en la produción y desarollo y por la ideología patriarcal sexista persistente en la sociedad, por tanto, es muy difícil que alcance el amparo de la ley; "de modo que a quien se dedica al trabajo del hogar y al cuidado de los hijos sólo se le reconocen obligaciones." (PAREJA, 1986)

Como se observa las políticas y planes de salud hasta aquel entonces eran referidos, direccionados y condicionados por reinvindicaciones de la clase trabajadora. Las acciones de salud continuaron siendo escasas, específicas y totalmente centralizadas.

MARTOS, M.V. E. P. de; FONSECA, R.M.G.S. da Gender and woman's health politics in Latino America: the case of the Peru. Rev.Esc.Enf.USP, v.30, n.1, p.44-57,Apr. 1996.

This study includes a presentation and analysis about the woman's problematic. It considers the woman social condition concording to the androgynors ideology of the society. Briefly it presents a historical evolution of the woman in accord with the gender analysis. On the other hand, it presents too, an analysis of the health and the public politics in relation to the woman in Latin America, mainly in Peru, since the Incanato period, justifying the motives of the formulation and implementation of the Family Planning Program, the Responsable Paternity Program and Child and Mother Atention Program.

UNITERMS: Ideology. Gender. Family planning. Health politics. 


\section{REFERÊNCIAS BIBLIOGRÁFICAS}

ARANÍBAR, C. El princípio de la dominacíon (1531-1580). In: LUMBRERAS, L.G. et al Nueva Historia General del Perú. Lima, Mosca Azul, s/d. 263p.

BARROSO, C. A saúde da mulher no Brasil. São Paulo, Conselho Estadual da Condição Feminina, 1985. 94p.

BARSTED, L.A.L. Legalização e descriminalização do aborto no Brasil - 10 anos de luta feminina. Estudos Feministas, n.2, p.104-130, 1992.

BEAUVOIR, S. O segundo sexo. $2^{a}$ ed. Rio de Janeiro, 1981.

BERTOLOZZI, M.R.; EGRY, E.Y.; SHIMA, H. Projeto USP/BID: Relatório de visita ao Peru. São Paulo, 1990. 40p./ mimeografado.

BLACK, M. Planificación de la familia - componente de la salud de la familia. In: CONFERENCIA INTERNACIONAL SOBRE LA CONTRIBUICIÓN DE LA PLANIFICACIÓN FAMILIAR A LA SALUD DE LA MUJER Y EL NIÑO, Nairobi, 1989. Anais. Nairobi, 1989, p.3-37

BRETAS, A.C.P. et al Política de Planejamento Familiar no Brasil: um problema de saúde pública. Acta Paul. Enf., v.3, n.2, p.59-63, 1990

BRONFMAN, M.P.; CASTRO, R.P. Discurso y práctica de la planificación familiar en America Latina. Saúde em Debate, n.34, p.61-68, 1989

BUNCH, C. Feminismo, democracia e direitos humanos. São Paulo, s/d/mimeografado.

CARDACI, D. Pensando como mujeres. Invest. y Educ. en Enferm., v.10, n.1, p.55-67, 1992.

CASTELLO, J. La atención de salud materno infantil en el Perú. Lima, 1985. 59p. / mimeografado

COSTA, A.O. et al Gênero e Universidade. São Paulo, Núcleo de Estudos da Mulher e Relações Sociais de Gênero, 1992. 176p.

DALLARI, S.G. O direito à saúde. Rev. Saúde Públ., v.22, n.1, p.57-63, 1988

DORA, D.D. et al Violência de gênero como violação de Direitos Humanos no Brasil. São Paulo, CLADEM-Brasil, 1992. 15p./mimeografado.

ELGEGREN, F.R. La mujer y la salud. In: FLORES, E.B. Participación de la Mujer. Lima, UNIFE, 1986. Cap.4

FARJE, H.G.; GUTIERREZ, I.D. Programa peruano de capacitación para la salud reproductiva. Lima, 1985. 57p. 
FONSECA, R.M.G.S. Mulher, reprodução biológica e classe social: a compreensão do nexo coesivo através do estudo dialético do perfil reprodutivo de mulheres atendidas nas Unidades Básicas de Saúde. São Paulo, 1990. 336p. Tese (Doutorado), Escola de Enfermagem, Universidade de São Paulo.

KELLY, J. Women, history and theory. Chicago, University of Chicago Press, 1986. 163p.

LABRA, M.E. Mulher, saúde e sociedade no Brasil. São Paulo, 1989. 302p.

LEITE, C.L.P. Mulheres: muito além do teto de vidro. São Paulo, Atlas, 1994. 346p.

LOPES, M.J.M. O trabalho da enfermeira: nem público, nem privado, feminino, doméstico, desvalorizado. Rev. Bras. Enf., v.41, n.3/4, p.211-17, 1988.

LORENZ, O. Sexualidade da mulher antes do casamento - opinioes de alunas universitárias. Acta Paul. Enf., v.3, n.2, p.64-7, 1990.

MACHUCA, E.V. Identidad femenina: cuestionando y construyendo estereotipos. Lima DESCO, 1991. 68p.

MAMEDE, M.V. Saúde da mulher - visão feminista. Femina, v.17, n.11. p.904-6, 1989.

MARIATEGUI, J.C. Siete ensayos de interpretación de la realidad peruana. Lima, Amauta, 1984

MINISTERIO DE SALUD. Hacia un Sistema Nacional Regionalizado e Integrado de Salud: objetivos, políticas, metas. Gestión Gubernamental, 1990-1995. Lima, 1990. 38p.

MOLYNEUX, M. Movilización sin emancipación? Los intereses de la mujer, Estado y revolución en Nicaragua. Desarrollo y Sociedad, n. 15, p.179-195, 1984.

NASCIMENTO, E.R. Política de atenção à saúde da mulher no Brasil: historicamente a quem se destina? Rev. Baiana de Enf., v.5, n.1, p.79-86, 1992.

OLIVEIRA, M.A.C. A reprodução humana em uma sociedade de classes: estudo dialético da representação de um conjunto de enfermeiras. São Paulo, 1988. 242p. Dissertação (Mestrado). Escola de Enfermagem, Universidade de São Paulo.

ORGANIZACIÓN PANAMERICANA DE LA SALUD La mujer en la salud y el desarrollo. $1983,59 p$.

PAREJA, P. El sistema educativo. In: FLORES, E.B. Participación de la Mujer. Lima, UNIFE, 1986. Cap.3.

PINOTTI, J.A.; GOMES, E. A mulher e a legislação. In: saúde: por uma política de saúde no Brasil. p.1-12, 1988 A mulher e o seu direito à

QUIJANO. A. Imperialismo, clases sociales y Estado en el Perú (1890-1930). Lima, Mosca Azul, 1985. 89p.

ROLDAN, J. Perú: mito y realidad. $3^{a}$ ed. Lima, Calas, 1989. 246p. 
ROMERO, O.L. Socialización papeles (roles) de género e imagem de la mujer en los medios masivos: quien perciben los estereotipos difundidos? Série Documentos de Investigación Número 1. México, 1988. p.716-749

TEIXEIRA, C.F. Políticas de saúde no Brasil: situação atual e desafios estratégicos. Saúde em Debate, n.35, p.4-7, 1987.

VEGAS, E.L. Viacrusis del Pueblo Peruano. Montevideo, Coopren, 1989. 160p.

VERUCCI, F. A mulher e o direito. São Paulo, Nobel, 1987. 211p.

WRIGHT, M.G.M.; CARNEIRO, A. O espaço da mulher brasileira e o espaço da enfermeira brasileira. Rev. Gaúcha de Enf., v.5, n.2, p.341-356, 1984. 